\title{
Genome analysis of Campylobacter jejuni strains isolated from a waterborne outbreak
}

\author{
Joana Revez ${ }^{1}$, Ann-Katrin Llarena' ${ }^{1}$, Thomas Schott ${ }^{1,2}$, Markku Kuusi ${ }^{3}$, Marjaana Hakkinen ${ }^{4}$, Rauni Kivistö ${ }^{1}$, \\ Marja-Liisa Hänninen ${ }^{1}$ and Mirko Rossi ${ }^{1 *}$
}

\begin{abstract}
Background: Waterborne Campylobacter jejuni outbreaks are common in the Nordic countries, and PFGE (pulsed field gel electrophoresis) remains the genotyping method of choice in outbreak investigations. However, PFGE cannot assess the clonal relationship between isolates, leading to difficulties in molecular epidemiological investigations. Here, we explored the applicability of whole genome sequencing to outbreak investigation by re-analysing three C. jejuni strains (one isolated from water and two from patients) from an earlier resolved Finnish waterborne outbreak from the year 2000.
\end{abstract}

Results: One of the patient strains had the same PFGE profile, as well as an identical overall gene synteny and three polymorphisms in comparison with the water strain. However, the other patient isolate, which showed only minor differences in the PFGE pattern relative to the water strain, harboured several polymorphisms as well as rearrangements in the integrated element CJIE2. We reconstructed the genealogy of these strains with ClonalFrame including in the analysis four C. jejuni isolated from chicken in 2012 having the same PFGE profile and sequence type as the outbreak strains. The three outbreak strains exhibited a paraphyletic relationship, implying that the drinking water from 2000 was probably contaminated with at least two different, but related, C. jejuni strains.

Conclusions: Our results emphasize the capability of whole genome sequencing to unambiguously resolve the clonal relationship between isolates of $C$. jejuni in an outbreak situation and evaluate the diversity of the C. jejuni population.

Keywords: Waterborne outbreak, Campylobacteriosis, Campylobacter jejuni, Whole genome sequencing, PFGE, SNP, Phage, Integrated element, Microevolution

\section{Background}

Campylobacter spp. are recognized as the leading cause of human bacterial gastroenteritis in the industrialized world [1]. In the European Union (EU), the incidence of human campylobacteriosis cases has followed an increasing trend in recent times and it continues to be the most commonly reported zoonosis with 214,268 confirmed cases in 2012 [2]. The majority of the infections are sporadic and seasonal, with a clear incidence peak in the summer months and early autumn [1]. Although infrequently reported compared with sporadic cases, outbreaks of campylobacteriosis do occur and are often associated with the consumption of raw milk and

\footnotetext{
*Correspondence: mirko.rossi@helsinki.fi

'Department of Food Hygiene and Environmental Health, Faculty of Veterinary Medicine, University of Helsinki, P.O. Box 66, (Agnes Sjöberginkatu 2), Helsinki Fl-00014, Finland

Full list of author information is available at the end of the article
}

contaminated drinking water [1-3]. In Finland, waterborne outbreaks caused by enteric pathogens are commonly registered [4-7] and C. jejuni was the causative agent in 19\% of the recorded outbreaks between 1998 and 2011 (http://www.thl.fi). This corresponds to approximately two C. jejuni waterborne outbreaks annually. Resolving $C$. jejuni outbreaks is complicated due to a prolonged lag time. A long incubation period (from 2 to 7 days) and lengthy diagnostic procedures cause an estimated lag time of approximately 2 weeks between time of exposure and recognition of the waterborne transmission [8]. This lag time may hinder the ability to detect $C$. jejuni from the water source, especially if the drinking water was transiently contaminated $[8,9]$.

PFGE (pulsed-field gel electrophoresis) typing of isolates has been widely used in outbreak investigations. PFGE is considered to be the gold standard for source tracking [8] due to the reported stability of PFGE genotypes 
in different host populations (e.g. human and chicken), irrespective of temporal and geographical space [10-12]. However, PFGE profiles cannot conclusively establish the clonal relationship between isolates, affecting the epidemiological investigations. Bacterial strains with identical PFGE or highly similar profiles isolated years apart generally show genetic diversity accumulated by genetic drift, homologous recombination or horizontal gene transfer $[10,13]$. On the contrary, the genomic differences between epidemiologically linked isolates sharing PFGE profiles are expected to be minor since the strains are considered to be the recent expansion of a single clone [14]. However, due to limited resolution capacity similar PFGE profiles could overestimate the clonal relationship between isolates [15,16]. Furthermore, since alterations in the PFGE patterns can result from a single genetic event due to a single-nucleotide polymorphism in a restriction site [9], bacteriophage acquisition or loss or transposition $[17,18]$, a clonal relationship may exist even between strains with different PFGE profiles [9].

Whole-genome sequencing (WGS) has recently been utilized to increase resolution power in the analysis of outbreak-associated isolates, leading to faster and more precise source identification in outbreak investigations, and to discriminate between alternative epidemiological hypotheses $[16,19]$. The aim of this study was to explore the applicability of WGS to an outbreak investigation by comparing the genomes of $C$. jejuni isolates from a Finnish waterborne outbreak that had occurred in 2000. The outbreak had already been resolved using both epidemiological and environmental analysis tools. All isolates have Penner serotype 12 and their KpnI and SacII profiles were identical, except for one patient isolate that had a three-band difference in the KpnI profile and a two-band difference in the SacII profile [4].

\section{Results and discussion}

Genome of $C$. jejuni water isolate 4031 and identification of mobile genetic elements

The combination of paired-end and $5 \mathrm{~kb}$ mate-pair library allowed the complete assembly of the genome of C. jejuni strain 4031, consisting of a single chromosome of $1,669,329$ nucleotides. Plasmid DNA was not detected. A total of 1,697 coding DNA sequences (CDSs) were identified in a coding area of $94.28 \%$ and function was predicted for $\sim 73 \%$. The strain belongs to ST-45 and, as previously observed in certain strains of the ST-45 complex, gamma-glutamyl transpeptidase, fucose permease and a secreted L-asparaginase were not detected [20]. The lipooligosaccharide (LOS) locus resembles class $\mathrm{P}$ of $C$. jejuni GB4 [21,22], which is associated with a non-sialylated LOS outer core structure without ganglioside mimicry [22]. IslandViewer predicted the presence of a putative prophage of 36,567 bp (from 441,523 bp to $478,090 \mathrm{bp}$ ) integrated between locus BN867_04520 (translation elongation factor G, homologue to Cj0493 of C. jejuni NCTC 11168) and locus BN867_05040 (hypothetical protein, homologue to Cj0494). This prophage showed $85.6 \%$ global nucleotide identity with the integrated element CJIE2 identified in $C$. jejuni RM1221 (calculated using Needleman-Wunsch global alignment algorithm) and it is integrated in the same region of the chromosome. The CJIE2 element in C. jejuni 4031 includes 51 open reading frames (ORFs), but a putative function was predicted for only three of these (BN867_04720, endonuclease; BN867_04740, phage repressor protein; BN867_04810, terminase B protein). Upon manual inspection, a second region that probably also has a phage origin was detected to be inserted between nucleotide 665,673 (corresponding to locus BN867_06900) and 670,480 (corresponding to locus BN867_06990). Pairwise comparison with $C$. jejuni RM1221 revealed that this region corresponds to a vestigial $\mathrm{Mu}$-like phage of approximately 4,807 bp (CJIE1; CJE0213-CJE0275): BN867_06900 is a homologue to CJE0275, and BN867_06990 is a homologue to CJE0213. The vestigial $\mathrm{Mu}$-like phage of $C$. jejuni 4031 is integrated in a different region than in C. jejuni RM1221: it is located upstream to the invasion phenotypic protein (BN867_0700/ BN867_0710; cipA). A vestigial Mu-like phage integrated upstream of $\operatorname{cip} A$ is also present in the genome of $C$. jejuni M1 (ST-137, ST-45 complex).

\section{The human outbreak-associated isolate IHV116292 underwent genome rearrangement}

The human C. jejuni isolates IHV116260 and IHV116292 were sequenced using paired-end library, assembled and mapped against $C$. jejuni 4031.

The KpnI PFGE profile of the human isolate IHV116292 differed from that of C. jejuni 4031 and IHV116060 by three bands [4], which were interpreted to mean that the isolate was closely related to the outbreak strain [23]. However, the assembled contigs of IHV116292 did not map unequivocally to the genome of $C$. jejuni 4031. On the contrary, all contigs of $C$. jejuni IHV116260 mapped completely to the water isolate genome.

Initial comparison between the outbreak isolates as well as other available genomes of $C$. jejuni strains belonging to the ST-45 was performed using BLASTN with default parameters and an atlas was built using C. jejuni 4031 as the reference genome (Figure 1). The more divergent genomic regions of all analysed ST-45 complex genomes (4031, IHV116260, IHV116292, BIGS0004, 55037, 4028, M1, 327) included the LOS, flagella and capsule (CPS) loci, as well as CJIE2 which was only present in the three outbreak isolates. As expected, the genomes of human isolates were highly similar to the reference genome of the water isolate. However, significant variation was detected 


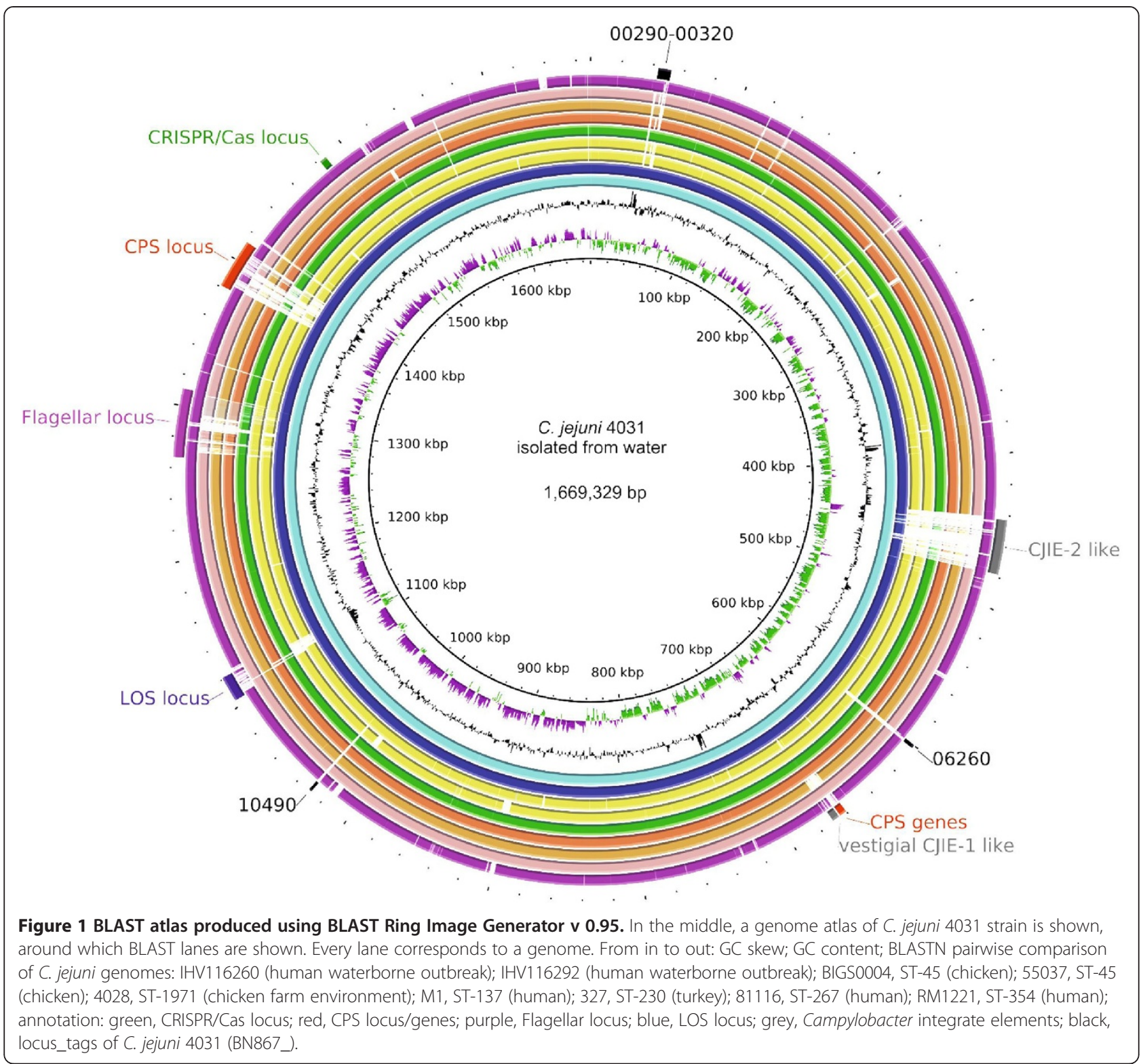

within the CJIE2 region between the isolates IHV116292 and 4031.

A collinear BLASTN comparison of the CJIE2 elements and the up- and downstream adjacent regions is shown in Figure 2. The CJIE2 of IHV116292 is 37,058 bp in length, includes 49 ORFs and shows $84.3 \%$ global nucleotide identity with CJIE2 of C. jejuni 4031 (calculated using Needleman-Wunsch global alignment algorithm) with the most divergent part located in the central $22 \mathrm{~kb}$ region. In addition, the CJIE2 of the genome of C. jejuni IHV116292 possesses a KpnI restriction site (in the gene immediately upstream of the Cj0594 homologue) which is not present in the CJIE2 of $C$. jejuni 4031. This additional restriction site explains the differences detected in KpnI patterns of IHV116292 (Figure 3).
C. jejuni 4031 and IHV116260 differ also from IHV116292 by an insertion of approximately $6 \mathrm{~kb}$ downstream of CJIE2, located between tRNA-2-selenouride synthase $(y b b B)$ and ferrochetolase (hemH). A second copy of this locus occurs downstream the Acyl-CoA thioester hydrolase (Cj0965c of C. jejuni NCTC 11168) and it is a homologue to the locus Cj0967-Cj0975 of C. jejuni NCTC 11168. The Cj0967-Cj0975 locus downstream of Cj0965c, as observed in IHV116292, is conserved among several $C$. jejuni strains including ST-45. By contrast, the second copy of this locus inserted between $y b b B$ and hemH has been previously described only in C. jejuni 81-176 [24] and BLASTN analysis showed that it is present also in C. jejuni M1 (Figure 2), 81116 and ICDCCJ07001 (data not shown). As described for C. jejuni 81-176 [24], both these loci in 


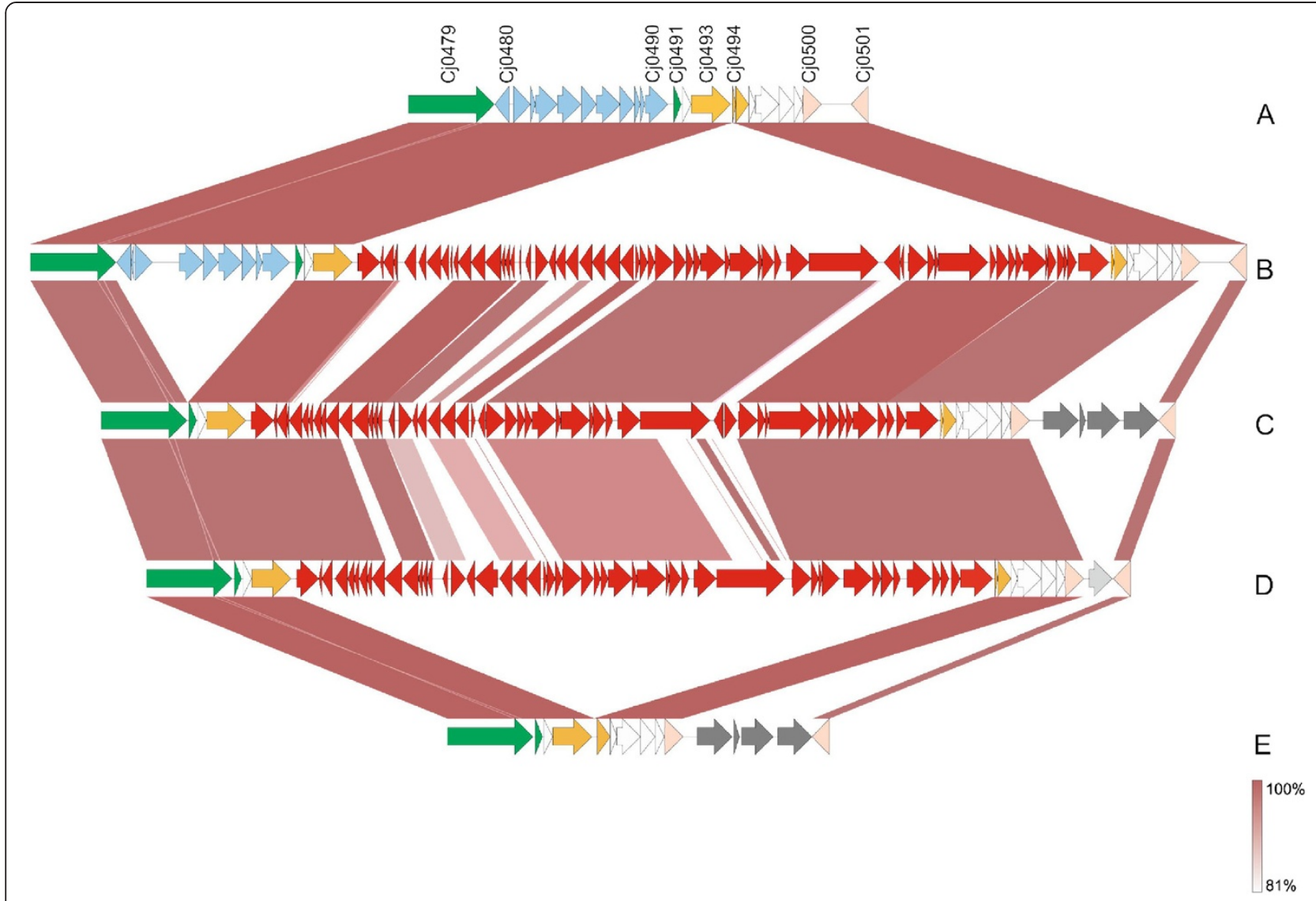

Figure 2 A collinear BLASTN comparison of the CJIE2 elements and the up- and downstream adjacent regions of $C$. jejuni strains (B) RM1221, (C) IHV116292, (D) 4031 with the corresponding region in C. jejuni strain (A) NCTC 11168 and (E) M1. Colour scheme: red, CJIE2; blue, fucose locus; dark grey, Cj0967-Cj0975-homolog cluster; green, conserved genes up- and downstream the fucose cluster; orange, conserved genes up- and downstream the CJIE2; pink, conserved genes up- and downstream the Cj0967-Cj0975-homolog cluster; grey, ammonium transporter.

C. jejuni 4031 harbour a $\sim 200$ bp specific region of intergenic AT-rich DNA, including an inverted repeat and a G-rich tract upstream the start codon of the Cj0967 homologue. In the $C$. jejuni isolate IHV116292 the locus downstream of $y b b B$ is substituted by a gene encoding an ammonium transporter showing $92.2 \%$ nucleotide identity with the pseudogene Cj0501 in C. jejuni NCTC 11168 and high amino acid identity with several $C$. jejuni and $C$. coli ammonium transporters. This gene is not present in the genome of either C. jejuni 4031 or IHV116260.

The analysis revealed that the human isolate IHV116292 contained several genome rearrangements inside and

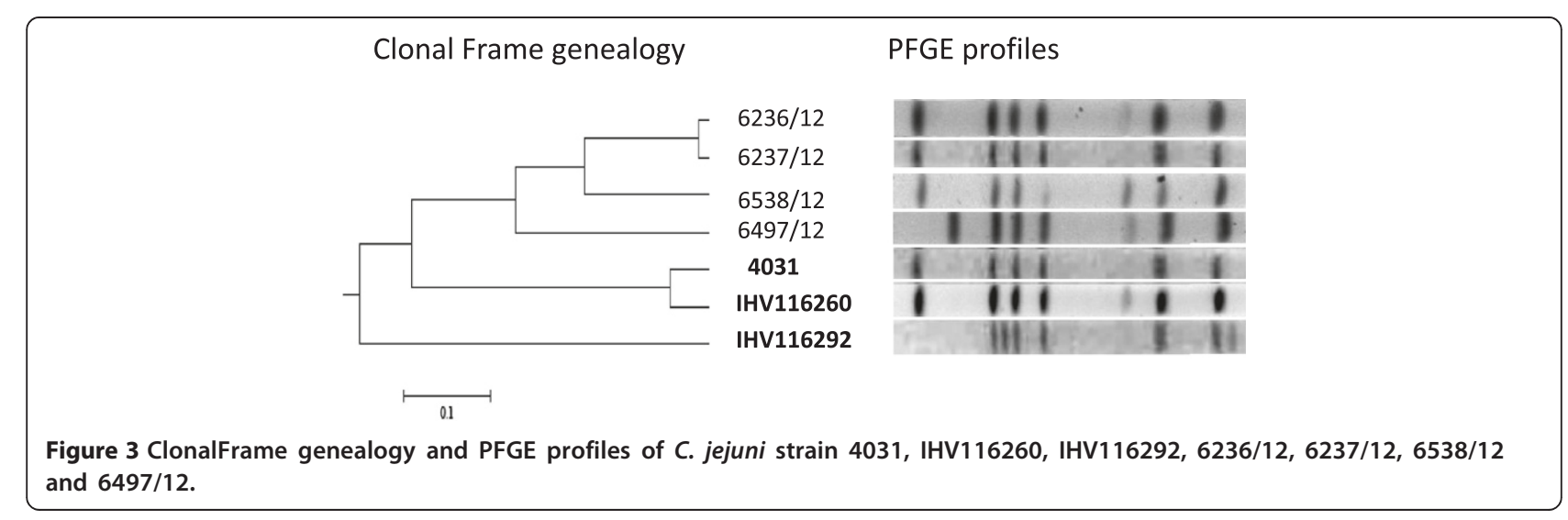


immediately downstream the CJIE2 element compared to the water isolate, and these were responsible for the observed differences in PFGE profiles. These differences were verified by PCR, thus they were not a consequence of misassembly or sequencing error. However, based on these data it cannot be determined whether these alterations have evolved in IHV116292 during the infection in the patient, or were already present in the population that caused the outbreak.

\section{SNP analysis and genealogy reconstruction suggest that C. jejuni 4031 and IHV116292 are two different strains}

To understand the microevolution of the waterborne outbreak-associated isolates, we analysed the polymorphic sites detected using progressiveMauve aligner. The polymorphic sites were checked manually if they fulfilled our criteria and they were divided into isolated SNPs and CNPs (see Methods). SNPs are most likely caused by mutations, whereas CNPs are probably the result of homologous recombination [25]. The human IHV116260 strain showed only three SNPs compared with the water strain 4031 ( $\mathrm{T} \rightarrow \mathrm{C}$ 143820, methyl-accepting chemotaxis signal transduction protein BN867_01350/BN867_01360; G $\rightarrow$ T, CJIE2 terminase B protein, BN867_04810; $\mathrm{C} \rightarrow \mathrm{G}$, LOS locus BN867_11290). In contrast, even when excluding the previously recognized variable regions (CJIE2 region and the Cj0967-Cj0975 duplicated locus downstream of $y b b B$ ), the human strain IHV116292 differed from $C$. jejuni 4031 by 69 SNPs, which are spread across the chromosome (see Additional file 1: Table S1). Furthermore, IHV116292 showed the presence of 9 CNPs affecting a total of 8 genes (see Additional file 1: Table S2).

Only a few SNPs are expected to be produced during a single passage through the host, based on previous studies in a human patient [26] and animal models [27,28]. The few differences observed between IHV116260 and 4031 can be a consequence of a single passage through the patient. By contrast, the much greater number of polymorphisms observed between IHV116292 and 4031 suggests that they were not generated during the outbreak.

To verify this hypothesis we attempted to reconstruct the genealogy of the three isolates. For this analysis we included the genomes of four additional ST-45 C. jejuni strains isolated from chicken 12 years after the outbreak. The chicken isolates had a KpnI PFGE profile indistinguishable from $C$. jejuni 4031 (6538/12; 6237/12; 6236/ 12) or differentiated by two bands (6497/12) (Figure 3). Two chicken strains $(6237 / 12 ; 6236 / 12)$ were obtained from different houses of the same farm, while the other two strains were obtained from two different farms two weeks later. It is expected that in the time frame of 12 years the isolates would accumulate several SNPs by genetic drift, allowing us to estimate the distance between C. jejuni 4031 and IHV116292, assuming that similar PFGE patterns originated from a common ancestor. The genealogy reconstructed using ClonalFrame based on core genome alignment obtained with progressiveMauve is presented in Figure 3. The tree shows a monophyletic relationship between $C$. jejuni 4031, the human isolate IHV116260 and the chicken strains. The $C$. jejuni human isolate IHV116292 is located in a separate branch that originates directly from the root. A BLASTN comparison of the genomes of C. jejuni 4031 and the chicken strains revealed that they are very similar (see Additional file 2: Figure S1). Differences between C. jejuni 4031 and the chicken strains were located in the CJIE2 (absent in strain 6497/12), the LOS locus and the flagellar locus. To estimate the genetic distance between the chicken and the outbreak strains, we compared the allelic profiles of 1,287 genes obtained from the PubMLST-Campylobacter database. Split decomposition (see Additional file 2: Figure S2) showed that the chicken strains are closer to C. jejuni 4031 and the human isolate IHV116260 (average distance of 0.0061 ) than IHV116292 (average distance of 0.0175). We further calculated the number of SNPs present between C. jejuni 4031 and the chicken strain 6236/12 using the same criteria applied for the outbreak strains. In particular, we excluded all regions that could be affected by homologous recombination (CNPs, CJIE2) in order to detect only those polymorphisms most likely acquired by mutation. From an original list of $\sim 1000$ polymorphisms extracted from progressive Mauve alignment of C. jejuni 4031 and 6236/12, only 64 SNPs fulfilled our criteria (see Additional file 1: Table S3). In fact, the majority of the polymorphisms were located within the CJIE2 or were classified as CNPs, indicating that homologous recombination explained the accumulation of genetic differences between C. jejuni 4031 and 6236/12.

These results suggest that the number of differences observed between C. jejuni 4031 and IHV116292 is too large for accumulation over the course of the outbreak. On the basis of the results, two hypotheses can be formulated: 1) the water was contaminated by a mixture of at least two related C. jejuni strains and 2) IHV116292 is not associated with the outbreak. Considering the findings of the epidemiological investigation [4], the first hypothesis appears to be the most plausible.

\section{Recombination is the probable origin of the observed differences in CJIE2}

CJIEs are postulated to be hypervariable genomic regions that contribute to diversity of C. jejuni [29]. In particular, CJIE1 (the Mu-like phage) has been shown to form a family of prophages with both conserved and divergent sequence regions, and appears to be adapted to $C$. jejuni [30]. Our analysis of three outbreak isolates showed that CJIE2 is variable even between highly related C. jejuni 
strains (Figure 2). We extended the comparison to include CJIE2 sequences of chicken strains of the same ST-45. Two different CJIE2 sequences were detected: 6236/12 and $6237 / 12$ have an identical CJIE2 sequence, whereas $6538 / 12$ possesses a CJIE2 that differs from the CJIE2 of $6236 / 12$ and $6237 / 12$ at three positions (see Additional file 2: Figure S3). Comparing the chickenassociated CJIE2 sequences with those of the outbreak strains, we observed that they are more related to CJIE2 of C. jejuni IHV116292 than to 4031, in spite of the ancestral relationship between the later and the chicken strains. This observation suggests that CJIE2 undergoes extensive recombination and genetic rearrangement, comparable with that of the Mu-like phage CJIE1. Considering that the endonucleases encoded by these elements inhibit natural transformation of C. jejuni [31], their hypervariability might influence the microevolution of closely related $C$. jejuni strains.

\section{Conclusions}

Outbreak strains are isolates that are both epidemiologically (e.g., by time, site and common source) and genetically related (i.e. have indistinguishable genotypes). Such isolates are presumed to be clonal [23]. However, in waterborne outbreaks, several varieties of pathogens (e.g. viruses, protozoa and bacteria) or a mixture of strains are sometimes detected in the water as well as in human samples as a result of waste water contamination [2]. In such cases several different outbreak-associated strains may be detected [8]. In the waterborne outbreak re-investigated in this study, two human isolates were attributed to the water contamination, based on serotype and PFGE data [4]. Although one of the human isolates had a slightly deviant PFGE pattern, this was not considered significant enough to exclude it from the outbreak, as a PFGE profile can change after only a single passage through the chicken host [32], by genomic rearrangement due to phage infection [17] or mobilization of temperate phages [18]. The PFGE pattern differences observed in IHV116292 could potentially have occurred during the passage though the human host. However, our comparative genomic analysis clearly reveals that this human isolate contains so many genomic alterations compared to the water strain, that it represents another $C$. jejuni strain. In this particular case, whole genome analysis was required to correctly define the clonal frame. This study highlights the capability of whole-genome sequencing to unambiguously resolve the relationship between the isolates of a $C$. jejuni outbreak. In the future, next-generation sequencing technologies will more intensively be applied as a tool for outbreak strain characterization, remarkable improving the reliability of epidemiological conclusions on the association between source and infected patients.

\section{Methods}

Bacterial strains, PFGE and DNA isolation

Two waterborne outbreak-associated C. jejuni isolates collected from two patients (IHV116292 and IHV116260) and one isolate from contaminated tap water (4031) were selected. All of the C. jejuni isolates were collected in August 2000 during a large outbreak of gastroenteritis that had occurred in a community in southern Finland $[4,8]$. This study was part of the public health response to a waterborne outbreak. According to Finnish legislation, no ethical approval is needed for this type of response. In addition, four chicken strains (6538/12, 6237/12, 6236/12 and 6497/12) isolated during summer 2012 over the course of the national Campylobacter monitoring programme were included. The strains were selected on the basis of their PFGE profile similarity to the outbreak isolates and having the same Multi Locus Sequence Type (MLST). The $K p n I$ PFGE patterns for the strains were produced as previously described [8]. High quality genomic DNA was isolated with the Wizard Genomic DNA Purification Kit (Promega, Germany), according to the manufacturer's instructions.

\section{Genome sequencing, assembly and annotation}

Genome sequences were obtained using Illumina sequencing technology with 100 cycles paired-end reads. In addition, a $5 \mathrm{~kb}$ mate-paired end library was performed for the isolate 4031. Illumina reads were trimmed using the Condetri perl script [33] with default settings, with a minimum read length of 75 nucleotides. All reads were assembled separately using MIRA [34,35] and ABySS [36]. The genomes of C. jejuni strains 4031, IHV116260 and IHV116292 were closed, and for the chicken strains virtual genomes were generated. For this purpose, the water isolate $C$. jejuni 4031 was used as a scaffold, and the contigs were re-ordered using Mauve [37]. Primary annotation of all strains was performed using Rapid Annotation using Subsystem Technology (RAST) [38], and later, the sequences were manually curated using Artemis [39]. Prophages were searched in the genomes using IslandViewer $[40,41]$. Clusters of hypothetical genes, generally associated with genomic islands [41], were searched and manually inspected.

\section{Comparative genomics}

Genomes 4031 (water sample from waterborne outbreak, this study), IHV116260 (human waterborne outbreak, this study), IHV116292 (human waterborne outbreak, this study), BIGS0004, ST-45 (chicken; NCBI ANGO), 55037, ST-45 (chicken; NCBI AIOH01), 4028, ST-1971 (chicken farm environment; ENA PRJEB6225), M1, ST-137 (human; NCBI NC_017280), 327, ST-230 (turkey; NCBI ADHM01), 81116, ST-267 (human; NCBI NC_009839), RM1221, ST-354 (human; NCBI NC_003912) were compared using 
BLAST and the atlases were generated using BLAST Ring Image Generator v 0.95 (BRIG; [42]). Synteny was evaluated using Mauve [43] and Artemis Comparative Tool (ACT; [44]). Linear comparison of integrated elements was performed using EasyFig v2.1 [45]. Assembled data were uploaded on usmirror1.pubmlst. org/campylobacter/ database and implemented with the Bacterial Isolate Genome Sequence Database (BIGS-DB) software [46] and allelic profiles for all common loci were retrieved. Allelic profiles of all isolates were compared using Splitstree4 [47]. Lists of polymorphisms were exported from pairwise analyses performed using Mauve and then curated manually. Polymorphisms were filtered to remove those likely due to assembly or alignment errors. Polymorphisms were filtered if i) detected in or immediately adjacent to the ribosomal operon, ii) the 50 nucleotides surrounding the polymorphism were not unique in the genome (analysed by BLASTN) or iii) detected in a homopolymeric run. Nucleotide polymorphisms were divided in isolated single nucleotide polymorphisms (SNPs) and clusters of nucleotide polymorphisms (CNPs). SNPs were defined as polymorphisms separated from the next nucleotide polymorphism by a difference greater than $200 \mathrm{bp}$ on both sides. CNPs were defined as groups of at least two polymorphisms with a distance of less than $200 \mathrm{bp}$ between two consecutive polymorphic sites, separated from the next sequence by a difference greater than $200 \mathrm{bp}$ on both sides. The clonal genealogy of the strains based on the whole genome was estimated using a model-based approach to determine bacterial microevolution implemented in ClonalFrame [48]. Genomes were aligned using progressiveMauve [43] and collinear blocks bigger than $500 \mathrm{bp}$ were filtered using the perl script stripSubsetLCBs available in the ClonalOrigin package [49]. Thus, ClonalFrame was run with 10,000 burn-in iterations followed by 10,000 data collection iterations. The consensus tree represents combined data from three independent runs, with $75 \%$ consensus required for inference of relatedness.

\section{Data deposition}

The genome of $C$. jejuni 4031 was submitted to EMBL with accession number HG428754. The sequence reads of the other strains were submitted to EMBL under project number PRJEB4165 (ERP003426).

\section{Additional files}

Additional file 1: Table S1. List of single nucleotide polymorphisms (SNPs) detected in C. jejuni IHV116292 compared with the reference strain C. jejuni 4031; Table S2. List of cluster of nucleotide polymorphisms (CNPs) detected in C. jejuni IHV116292 compared with the reference strain C. jejuni 4031; Table S3. List of single nucleotide polymorphisms (SNPs) detected in C. jejuni 6236/12 compared with the reference strain C. jejuni 4031.
Additional file 2: Figure S1. BLAST atlas produced using BLAST Ring Image Generator v 0.95 comparing C. jejuni 4031 strain versus $C$. jejuni HV1 16260, IHV1 16292, 6497/12, 6538/12, 6236/12, 6237/12; Figure S2. (A) Split decomposition and (B) Distance matrix of the allelic profiles of 1,287 genes obtained from PubMLST-Campylobacter database of $C$. jejuni strains 4031, HV1 16260, IHV116292, 6497/12, 6538/12, 6236/12 and $6237 / 12$; Figure S3. A collinear BLASTN comparison of the CJIE2 elements of C. jejuni strains RM1221, 4031, IHV1 16292, 6538/12 and 6236/12.

\section{Competing interests}

The authors declare that they have no competing interests.

\section{Authors' contributions}

JR participated in the design of the study, genomic analysis and drafted the manuscript. AKL carried out experimental work and data collection, and helped draft the manuscript. TS performed the genome assembly and helped draft the manuscript. MK and MH contributed to the data acquisition. RK participated in the study design. MLH participated in the design of the study and coordination, and helped draft the manuscript. MR conceived the study, participated in the study design and coordination, was responsible for the comparative genome analysis and drafted the manuscript. All authors read and approved the final manuscript.

\section{Acknowledgments}

We thank Urszula Hirvi for her excellent technical assistance. This work was supported by the Academy of Finland grant on behalf of CoE-MiFoSa (n. 11411405).

\section{Author details}

'Department of Food Hygiene and Environmental Health, Faculty of Veterinary Medicine, University of Helsinki, P.O. Box 66, (Agnes Sjöberginkatu 2), Helsinki Fl-00014, Finland. ${ }^{2}$ Biology Oceanography, Leibniz Institute for Baltic Sea Research, Rostock-Warnemünde, Germany. ${ }^{3}$ National Institute for Health and Welfare, Helsinki, Finland. ${ }^{4}$ Research Department, Finnish Food Safety Authority, Helsinki, Finland.

Received: 5 November 2013 Accepted: 5 September 2014 Published: 8 September 2014

\section{References}

1. Olson CK, Ethelberg S, Pelt W, Tauxe RV: Epidemiology of Campylobacter jejuni infections in industrialized nations. In Campylobacter. Edited by Nachamkin I, Szymanski CM, Blaser MJ. Washington DC, USA: American Society for Microbiology; 2008:163-189.

2. European Food Safety Authority (EFSA), European Centre for Disease Prevention and Control (ECDC): The European Union Summary Report on Trends and Sources of Zoonoses, Zoonotic Agents and Food-borne Outbreaks in 2012. EFSA Journal 2014, 12:3547.

3. Taylor EV, Herman KM, Ailes EC, Fitzgerald C, Yoder JS, Mahon BE, Tauxe RV: Common source outbreaks of Campylobacter infection in the USA, 1997-2008. Epidemiol Infect 2013, 141:987-996.

4. Kuusi M, Klemets P, Miettinen I, Laaksonen I, Sarkkinen H, Hanninen ML, Rautelin $\mathrm{H}$, Kela E, Nuorti JP: An outbreak of gastroenteritis from a non-chlorinated community water supply. J Epidemiol Community Health 2004, 58(4):273-277.

5. Kuusi M, Nuorti JP, Hänninen ML, Koskela M, Jussila V, Kela E, Miettinen I, Ruutu P: A large outbreak of campylobacteriosis associated with a municipal water supply in Finland. Epidemiol Infect 2005, 133(4):593-601.

6. Pitkanen T, Miettinen IT, Nakari UM, Takkinen J, Nieminen K, Siitonen A, Kuusi M, Holopainen A, Hanninen ML: Faecal contamination of a municipal drinking water distribution system in association with Campylobacter jejuni infections. J Water Health 2008, 6(3):365-376.

7. Schönberg-Norio D, Takkinen J, Hänninen ML, Katila ML, Kaukoranta SS, Mattila L, Rautelin H: Swimming and Campylobacter infections. Emerg Infect Dis 2004, 10(8):1474-1477.

8. Haajanen H, Pummi T, Wermundsen K, Katila ML, Sarkkinen H, Miettinen I, Rautelin H: Detection and typing of Campylobacter jejuni and Campylobacter coli and analysis of indicator organisms in three waterborne outbreaks in Finland. Appl Environ Microbiol 2003, 69(3):1391-1396. 
9. On SL, McCarthy N, Miller WG, Gilpin BJ: Molecular epidemiology of Campylobacter species. In Campylobacter. 3rd edition. Edited by Nachamkin I, Szymanski CM, Blaser MJ. Washington, DC: ASM Press, American Society for Microbiology; 2008:191-211

10. Hänninen ML, Perko-Makela P, Rautelin H, Duim B, Wagenaar JA: Genomic relatedness within five common Finnish Campylobacter jejuni pulsedfield gel electrophoresis genotypes studied by amplified fragment length polymorphism analysis, ribotyping, and serotyping. Appl Environ Microbiol 2001, 67(4):1581-1586

11. Williams A, Oyarzabal OA: Prevalence of Campylobacter spp. in skinless, boneless retail broiler meat from 2005 through 2011 in Alabama, USA. BMC Microbiol 2012, 12:2180. 12-184.

12. Laturnus $C$, Jores J, Moser I, Schwerk P, Wieler LH: Long-term clonal lineages within Campylobacter jejuni 0:2 strains from different geographical regions and hosts. Int J Med Microbiol 2005, 294(8):521-524

13. Orsi RH, Borowsky ML, Lauer P, Young SK, Nusbaum C, Galagan JE, Birren BW, Ivy RA, Sun Q, Graves LM, Swaminathan B, Wiedmann M: Short-term genome evolution of Listeria monocytogenes in a non-controlled environment. BMC Genomics 2008, 9:9-2164.

14. Li W, Raoult D, Fournier PE: Bacterial strain typing in the genomic era. FEMS Microbiol Rev 2009, 33(5):892-916.

15. Kennedy AD, Otto M, Braughton KR, Whitney AR, Chen L, Mathema B, Mediavilla JR, Byrne KA, Parkins LD, Tenover FC, Kreiswirth BN, Musser JM, DeLeo FR: Epidemic community-associated methicillin-resistant Staphylococcus aureus: recent clonal expansion and diversification. Proc Natl Acad Sci U S A 2008, 105(4):1327-1332.

16. Harrison EM, Paterson GK, Holden MT, Larsen J, Stegger M, Larsen AR, Petersen A, Skov RL, Christensen JM, Bak Zeuthen A, Heltberg O, Harris SR, Zadoks RN, Parkhill J, Peacock SJ, Holmes MA: Whole genome sequencing identifies zoonotic transmission of MRSA isolates with the novel mecA homologue mecC. EMBO Mol Med 2013, 5(4):509-515.

17. Scott AE, Timms AR, Connerton PL, Loc Carrillo C, Adzfa Radzum K, Connerton IF: Genome dynamics of Campylobacter jejuni in response to bacteriophage predation. PLoS Pathog 2007, 3(8):e119.

18. Barton C, Ng LK, Tyler SD, Clark CG: Temperate bacteriophages affect pulsed-field gel electrophoresis patterns of Campylobacter jejuni. J Clin Microbiol 2007, 45(2):386-391.

19. Lewis T, Loman NJ, Bingle L, Jumaa P, Weinstock GM, Mortiboy D, Pallen MJ: High-throughput whole-genome sequencing to dissect the epidemiology of Acinetobacter baumannii isolates from a hospital outbreak. J Hosp Infect 2010, 75(1):37-41.

20. de Haan CP, Llarena AK, Revez J, Hanninen ML: Association of Campylobacter jejuni metabolic traits with multilocus sequence types. Appl Environ Microbiol 2012, 78(16):5550-5554.

21. Parker CT, Gilbert M, Yuki N, Endtz HP, Mandrell RE: Characterization of lipooligosaccharide-biosynthetic loci of Campylobacter jejuni reveals new lipooligosaccharide classes: evidence of mosaic organizations. J Bacteriol 2008, 190(16):5681-5689

22. Godschalk $P C$, van Belkum $A$, van den Braak $N$, van Netten $D$, Ang CW, Jacobs BC, Gilbert M, Endtz HP: PCR-restriction fragment length polymorphism analysis of Campylobacter jejuni genes involved in lipooligosaccharide biosynthesis identifies putative molecular markers for Guillain-Barre syndrome. J Clin Microbiol 2007, 45(7):2316-2320.

23. Tenover FC, Arbeit RD, Goering RV, Mickelsen PA, Murray BE, Persing DH, Swaminathan B: Interpreting chromosomal DNA restriction patterns produced by pulsed-field gel electrophoresis: criteria for bacterial strain typing. J Clin Microbiol 1995, 33(9):2233-2239.

24. McLennan MK, Ringoir DD, Frirdich E, Svensson SL, Wells DH, Jarrell H, Szymanski CM, Gaynor EC: Campylobacter jejuni biofilms up-regulated in the absence of the stringent response utilize a calcofluor white-reactive polysaccharide. J Bacterio/ 2008, 190(3):1097-1107.

25. Kennemann L, Didelot X, Aebischer T, Kuhn S, Drescher B, Droege M, Reinhardt R, Correa P, Meyer TF, Josenhans C, Falush D, Suerbaum S: Helicobacter pylori genome evolution during human infection. Proc Natl Acad Sci U S A 2011, 108(12):5033-5038.

26. Revez J, Schott T, Llarena AK, Rossi M, Hänninen ML: Genetic heterogeneity of Campylobacter jejuni NCTC 11168 upon human infection. Infect Genet Evol 2013, 16:305-309.
27. Jerome JP, Bell JA, Plovanich-Jones AE, Barrick JE, Brown CT, Mansfield LS Standing genetic variation in contingency loci drives the rapid adaptation of Campylobacter jejuni to a novel host. PLoS One 2011, 6(1):e16399.

28. Kim JS, Artymovich KA, Hall DF, Smith EJ, Fulton R, Bell J, Dybas L, Mansfield LS, Tempelman R, Wilson DL, Linz JE: Passage of Campylobacter jejuni through the chicken reservoir or mice promotes phase variation in contingency genes Cj0045 and Cj0170 that strongly associates with colonization and disease in a mouse model. Microbiology 2012, 158(Pt 5):1304-1316.

29. Quiñones B, Guilhabert MR, Miller WG, Mandrell RE, Lastovica AJ, Parker CT: Comparative genomic analysis of clinical strains of Campylobacter jejuni from South Africa. PLoS One 2008, 3(4):e2015.

30. Clark CG, Ng LK: Sequence variability of Campylobacter temperate bacteriophages. BMC Microbio/ 2008, 8(8-49):49-2180.

31. Gaasbeek EJ, Wagenaar JA, Guilhabert MR, van Putten JP, Parker CT, van der Wal FJ: Nucleases encoded by the integrated elements CJIE2 and CJIE4 inhibit natural transformation of Campylobacter jejuni. J Bacteriol 2010, 192(4):936-941.

32. Hänninen ML, Hakkinen M, Rautelin H: Stability of related human and chicken Campylobacter jejuni genotypes after passage through chick intestine studied by pulsed-field gel electrophoresis. Appl Environ Microbiol 1999, 65(5):2272-2275.

33. Smeds L, Kunstner A: ConDeTri - a content dependent read trimmer for Illumina data. PLoS One 2011, 6(10):e26314.

34. Chevreux B, Wetter T, Suhai S: Chevreux B, Wetter T, Suhai S: Genome sequence assembly using trace signals and additional sequence information. Abstr German Conf Bioinform (Gcb) 1999, 99:45-56.

35. Chevreux B, Pfisterer T, Suhai S: Automatic assembly and editing of genomic sequences. In Genomics and proteomics - functional and computational aspects. Chap. 5th edition. Edited by Suhai S. New York: Kluwer Academic/Plenum Publishers; 2000:51-65.

36. Simpson JT, Wong K, Jackman SD, Schein JE, Jones SJ, Birol I: ABySS: a parallel assembler for short read sequence data. Genome Res 2009, 19(6):1117-1123.

37. Darling AC, Mau B, Blattner FR, Perna NT: Mauve: multiple alignment of conserved genomic sequence with rearrangements. Genome Res 2004 14(7):1394-1403.

38. Aziz RK, Bartels D, Best AA, DeJongh M, Disz T, Edwards RA, Formsma K, Gerdes S, Glass EM, Kubal M, Meyer F, Olsen GJ, Olson R, Osterman AL, Overbeek RA, McNeil LK, Paarmann D, Paczian T, Parrello B, Pusch GD, Reich C, Stevens R, Vassieva O, Vonstein V, Wilke A, Zagnitko O: The RAST Server: rapid annotations using subsystems technology. BMC Genomics 2008, 9:75.

39. Rutherford K, Parkhill J, Crook J, Horsnell T, Rice P, Rajandream MA, Barrell B: Artemis: sequence visualization and annotation. Bioinformatics 2000 16(10):944-945.

40. Langille MG, Brinkman FS: IslandViewer: an integrated interface for computational identification and visualization of genomic islands. Bioinformatics 2009, 25(5):664-665.

41. Hsiao WW, Ung K, Aeschliman D, Bryan J, Finlay BB, Brinkman FS: Evidence of a large novel gene pool associated with prokaryotic genomic islands. PLoS Genet 2005, 1(5):e62.

42. Alikhan NF, Petty NK, Ben Zakour NL, Beatson SA: BLAST Ring Image Generator (BRIG): simple prokaryote genome comparisons. BMC Genomics 2011, 12:402-2164. 12-402.

43. Darling AE, Mau B, Perna NT: progressiveMauve: multiple genome alignment with gene gain, loss and rearrangement. PLoS One 2010, 5(6):e11147.

44. Carver TJ, Rutherford KM, Berriman M, Rajandream MA, Barrell BG, Parkhill J: ACT: the Artemis Comparison Tool. Bioinformatics 2005, 21(16):3422-3423.

45. Sullivan MJ, Petty NK, Beatson SA: Easyfig: a genome comparison visualizer. Bioinformatics 2011, 27(7):1009-1010.

46. Jolley KA, Maiden MC: BIGSdb: Scalable analysis of bacterial genome variation at the population level. BMC Bioinformatics 2010, 11:595-2105. 11-595.

47. Huson DH, Bryant D: Application of phylogenetic networks in evolutionary studies. Mol Biol Evol 2006, 23(2):254-267.

48. Didelot $X$, Falush D: Inference of bacterial microevolution using multilocus sequence data. Genetics 2007, 175(3):1251-1266.

49. Didelot X, Lawson D, Darling A, Falush D: Inference of homologous recombination in bacteria using whole-genome sequences. Genetics 2010, 186(4):1435-1449.

\section{doi:10.1186/1471-2164-15-768}

Cite this article as: Revez et al: Genome analysis of Campylobacter jejuni strains isolated from a waterborne outbreak. BMC Genomics 2014 15:768. 